\title{
A virtual terminal program for data transfer between a Data General MicroNOVA laboratory computer and a DECsystem-10 timesharing system
}

\author{
WILLIAM H. GANOE and DAVID E. KIERAS \\ University of Arizona, Tucson, Arizona 85721
}

\begin{abstract}
A program for transferring data files between a small lab computer and a large timesharing system is described. The program is based on the concept of having the lab computer simulate a timesharing terminal. General features of the approach and the major implementation problems are discussed.
\end{abstract}

A laboratory computer, while specialized for collecting data, is usually not suited to performing routine statistical analysis of the same data. Such machines may have too small a word size for numerical accuracy, or they may have too small a memory capacity to allow the programming of statistical problems of any complexity. Furthermore, such programming often represents wasted effort, since powerful statistical packages are usually available on a large computer at the same institution. Hence, the most effective strategy is to collect the data on the small lab computer, but to analyze it on the local computer center's large machine. However, this strategy requires some method for conveying the data from the lab machine to the center's machine. A useful, though less common, activity consists of conveying data from the large machine to the lab computer. These activities require some communication medium or link between the two computers.

Perhaps the oldest method of communication involves some portable medium such as cards, paper tape, or magnetic tape, which is simply carried from one machine to the other. The major advantage of this method is its simplicity, together with the fact that a record in the medium may be desired anyway. However, card equipment is unduly expensive and slow, paper tape is fragile and often presents formatcompatibility problems, and magnetic tape both requires expensive equipment and often presents format-compatibility problems as well.

A more modern approach to transmitting data between two computers is the network approach, in which a direct hardware connection between the computers is used to transmit data at high speed. Once implemented, such a link is clearly the best

Requests for reprints should be sent to the first author at the Department of Psychology, University of Arizona, Tucson, Arizona 85721. solution. However, such a link can require the purchase of special hardware for both computers, and it requires complex programs both for the lab machine and for the large computer center machine to handle the linking hardware.

In setting up our computerized laboratory, we were faced with the communications problem. Our lab computer is a Data General MicroNoVA, running under Data General's diskette operating system (DOS). Although this machine is quite satisfactory for data collection, it suffers from only $28 \mathrm{~K}$ of memory and slow floppy disks, essentially eliminating any possibility of substantial amounts of statistical computing. In contrast, our computer center is an excellent installation, consisting of a DEC KL-10 timesharing system running under TOPS-10, with a hardware link to a CDC CYBER-175 that has all of the standard statistical packages. While a direct network connection between our lab machine and the DEC-10 was always a possibility, the implementation of DECNET protocol support is not yet completed, and it was still on the drawing boards at the time the lab machine installation was completed. To further complicate matters, the computer center was not equipped to read Data General format floppy disks, and we did not have the budget for magnetic tape, card, or paper tape equipment. Hence, to communicate our lab computer data to the computer center's machine required a different approach than either the portable medium or the network connection.

Our solution was to write a "virtual terminal" program for our lab computer. This is a program that makes the lab computer look like an ordinary computer terminal to the computer center's timesharing system. The transmission of data from the center's machine to the lab machine is achieved by simulating the listing of a file on a timesharing terminal. The transmission of data from the lab computer to the center's computer is done by simu- 
lating the building of a file from entry on the terminal keyboard. The hardware link is provided by an ordinary modem to send and receive data from the timesharing system. We use either a standard acoustic coupler modem at 300 baud for a dial-up connection or a modem on a hard-wired line running at 1,200 baud for higher speed communication.

This virtual terminal approach has four major advantages: (1) Complex programming had to be done for only one machine, the simpler laboratory computer. (2) We did not have to wait for the implementation of a general networking facility by the computer center but could start using our linking system as soon as we completed our own programming. (3) We did not have to invest in synchronous interface hardware that would be of little other use; the asynchronous interface we use for the virtual terminal link does double duty to drive a line printer. With a limited budget for hardware, this factor was especially important. (4) We can link with other DEC-10 systems that behave the same way. For example, the virtual terminal program can be used to communicate with DEC-10 systems on the ARPANET, as well as our local system. The lab computer thus provides a crude but effective way to communicate files between our computer center machine, which is not on the ARPANET, and machines that are.

The virtual terminal program transmits data considerably faster and more conveniently than could be done with the portable medium strategy. However, it is not as fast as a direct network link would be. More seriously, the virtual terminal approach cannot achieve the degree of error-free reliability that a true networking system would incorporate. In practice, however, this has not been a serious problem.

The remainder of this paper covers the general characteristics of our virtual terminal program, called LINK10, with emphasis on the general problems that had to be solved in order to have the program work reliably and at high transmission speeds. The program is written in extended assembler for the MicroNOVA and would be usable with little modification on any NOVA-line computer using DOS. However, it is hoped that the general strategies incorporated in our program will be useful to those with different machines who need to solve the linkage problem.

\section{DESCRIPTION OF THE PROGRAM}

LINK10 has two distinct modes of operation, terminal mode and file-transfer mode. In terminal mode, the MicroNOVA console looks like an ordinary terminal connected through phone lines to the DEC10. The only obvious difference is that the LINK10 program has to be invoked on the MicroNOVA before establishing contact with the DEC-10.

In file-transfer mode, there are actually two sub- modes, one for sending a file from the DEC-10 to the MicroNOVA and one for sending a file from the MicroNOVA to the DEC-10. The user goes from terminal mode to the desired file-transfer submode by typing a special control character on the MicroNOVA console. The program makes a graceful return to terminal mode when a file transfer is completed, when the user enters $\uparrow A$ or $\uparrow C$ at the console, or when an error occurs, such as running out of disk space. If an error occurs, an informative message is written on the console display.

\section{Terminal Mode}

Terminal mode is quite simple in concept. The LINK10 program merely waits for a character to come in from the console or the DEC-10. If the character comes from the DEC-10, it is sent to the console; if it comes from the console, it is sent to the DEC-10.

The implementation isn't quite that simple. Normal DEC-10 operation involves the frequent use of the break character, $\uparrow C$. However, under MicroNOVA DOS, $\uparrow C$ is also a break character, and if it is typed on the console, it causes immediate termination of the program and writing of a core dump to disk. Furthermore, if DOS is allowed to intercept the $\uparrow C$ character, the $\uparrow C$ will not be sent to the DEC-10, so the user will still be logged on and executing whatever program he attempted to halt with the $\uparrow C$. Since our users have a set of automatic reactions for dealing with the DEC-10, this situation is intolerable; the MicroNOVA DOS must not be allowed to respond to the $\uparrow C$ character. One possible solution would be to use the DOS system call for disabling console interrupts on break characters, but then the program would never see a $\uparrow A$ or $\uparrow C$, and some other character from the console would have to be substituted for the $\uparrow C$ function. Given the same set of users, this solution would merely substitute one frustrating characteristic for another. Another possible solution would be to disable all input interrupts from the console and have the program get console input by polling. This seemingly simplistic solution actually unduly complicates the rest of the program.

The solution chosen was to provide a new interrupt handler for the console. This handler could respond to special characters exactly as we wished it to, and DOS would never see any of them. The problem was how to get the system to use our console interrupt handler instead of its own. This was accomplished by switching pointers in the system interrupt handler dispatch table when LINK10 is started and restoring the original pointers before exiting. The information required for this kludge was obtained from a source listing of an early version of the DOS interrupt handler, imaginative use of the symbolic debugger, and a little horse sense. We would recommend buying and using a current source listing of the system 
interrupt handler. Under no circumstances do we recommend making permanent changes in the system software.

\section{MicroNOVA to DEC-10 Transfer}

Transferring a file from the MicroNOVA to the DEC-10 is fairly simple for the user and in implementation concept. The user types $\uparrow D<$ MicroNOVA file name $\rangle=\langle\mathrm{DEC}-10$ file name $\rangle\langle\mathrm{CR}\rangle$ then sits back and waits. The " $<\ldots$. " ' indicates a syntactic unit or a label for a nonalphanumeric character, rather than the actual characters used. When the $\langle\mathrm{CR}\rangle$ is typed, the MicroNOVA file is opened for reading, and the DEC-10 file name is inserted into a preset command string and the string is sent to the DEC-10. The form of the command string is COPY $\langle\mathrm{DEC}-10$ file name $\rangle=\mathrm{TTY}:\langle\mathrm{CR}\rangle$, which on the DEC-10 causes the file to be created and all input from the terminal to be copied to the file until an end-of-file character, $\uparrow Z$, is received. Then the MicroNOVA file is read in 256-character blocks and sent to the DEC-10 character by character. The characters are echoed by the DEC-10, and LINK 10 displays these echoed characters on the console to add to the user's peace of mind and to aid in debugging. When the end of the MicroNOVA file is detected, a $\uparrow Z$ is sent to the DEC-10, the MicroNOVA file is closed, and the program returns to terminal mode.

This description has glossed over a few big implementation problems. The DEC-10 has limited input buffers, which are adequate if a human is doing a hunt-and-peck routine on the terminal. However, if there is another computer shoving characters at 1,200 baud at the DEC-10 when it is fully loaded, some of the data can easily be lost. The DEC-10 sends $\uparrow$ Gs when its input buffers are full, but in practice, this control character was not sent soon enough to allow transmission to be halted quickly enough to avoid losing a few characters, especially when the DEC-10 was heavily loaded.

The solution that was finally chosen was for LINK10 to send a new character only after the previous one was echoed; hence the MicroNOVA only transmits as fast as the DEC-10 can acknowledge the characters. Furthermore, by waiting not just for any echoed character, but for the transmitted character, LINK10 can detect most transmission errors. This strategy reduces transmission speed to no more than half of the speed of the line, but this has proven to be only a minor annoyance.

The strategy of waiting for transmitted characters to be echoed had several pitfalls, since what the DEC-10 echoes is not always identical to what it is sent. In particular, the DEC-10 normally translates tabs to a series of blanks. So if a tab is sent the only characters echoed would be blanks. Since our terminals have no internal tab-handling facilities, some piece of software would have to translate the tabs to blanks for terminal mode. It seemed easiest to let the DEC-10 do the translation for terminal mode and then to tell the DEC-10 not to do the translation in file-transfer mode by setting the terminal characteristics with additional preset commands. Hence, before the above COPY command, the command SET TTY TAB $\angle C R>$ was issued, and after the end-of-file character, the command SET TTY NO TAB $<C R>$ was sent. A further problem with echoed characters is that the DEC-10 normally transmits fill characters in abundance; the LINK10 user thus needs to set the DEC-10 fill mode to NO FILL to eliminate these unwanted characters.

But it is still necessary to make allowance for extra characters, of an essentially indeterminate nature, appearing in the string of echoed characters. For example, the DEC-10 echoes $\langle\mathrm{CR}\rangle\langle\mathrm{LF}\rangle$ in response to $\mathrm{a}\langle\mathrm{CR}\rangle$. While each such extra character could be treated as a special case, a more satisfactory solution was to have the program count the number of unexpected characters echoed before the lasttransmitted one appears. A certain number of such unexpected echo characters are permitted; if more appear, the program concludes that a transmission error has occurred. This is a very general solution to the problem of dealing with the extra echo characters, and it has proved very useful in making file transfers to ARPANET machines, which have echoing conventions rather different from our own DEC-10.

In order to minimize overall transmission time, two buffers are maintained for outgoing characters. This allows the MicroNOVA system to be writing into the inactive buffer from disk while characters are being removed from the active buffer and sent to the DEC-10. Of course, since everything is interrupt driven, the data buffers have to have some sort of locks on them. The sections of code that access the active buffers are considered critical sections, and all interrupts are disabled while these sections are being executed.

\section{DEC-10 to MicroNOVA Transfer}

Transferring a file from the DEC-10 to the MicroNOVA is also easy for the user and conceptually quite simple. The user types $\uparrow N<D E C-10$ file name $>=$ $<$ MicroNOVA file name $><C R>$ and again sits back and waits. When the $\langle\mathrm{CR}\rangle$ is typed, first, the MicroNOVA file is created and opened for writing. It is considered an error if the file already exists. Then, the DEC-10 file name is inserted into a preset command string, and the string is sent to the DEC-10. This string has the form COPY TTY: $=\angle D E C-10$ file name $>\langle C R\rangle$, which causes the named file to be copied to the terminal. The incoming characters from the DEC-10 are copied to the MicroNOVA console and packed into 256-character buffers. When a 
buffer becomes full, a $\uparrow S$ control character is sent to the DEC-10 to suspend transmission, and the full buffer is written to the MicroNOVA disk. A $\uparrow Q$ is then sent to the DEC-10 to resume transmission. When the end of the DEC-10 file is detected, the MicroNOVA file is closed, and the program returns to terminal mode.

Again several problems were glossed over. A major problem is the possible loss of incoming characters when the buffer becomes full. The DEC10 may send a few characters between the time the $\uparrow S$ is sent and the time it is actually received and responded to by the DEC-10. This problem was solved by maintaining two buffers for incoming characters. As soon as one buffer fills up, the buffer pointers are set to the empty buffer, the $\uparrow S$ is sent, and then the system output routines are called for the full buffer. Thus there will always be a place to put incoming characters.

Detection of the end of the DEC-10 file is a bit sticky, because the COPY command does not generate a special end-of-file character. Rather, certain sequences of control characters such as , $\langle\mathrm{CR}>\langle\mathrm{LF}\rangle$ $<\mathrm{CR}><\mathrm{LF}\rangle$." or " $<\mathrm{CR}><\mathrm{LF}><\mathrm{CR}><\mathrm{DEL}\rangle$." are generated after the last line of the file. By writing LINK10 to recognize these patterns, file transfer is possible without the existence of a second program on the DEC-10, which simplified accessing other DEC-10 systems on the ARPANET. Although less satisfying than the use of an unambiguous end-of-file indication, this approach has generated no major problems in actual use.

Another problem is the handling of special character strings. One such case is the end-of-line pattern " $\angle \mathrm{CR}><\mathrm{LF}\rangle$." When this pattern appears, the <LF> should be sent to the console, but it should not be sent to the buffer for the MicroNOVA file. Another case is the detection of meaningful control characters (e.g., $\uparrow A, \uparrow C, \uparrow D$, and $\uparrow N$ ). The problem of detecting these patterns and special characters was solved by implementing finite automata and a fast means of classifying characters.

The recognition of character string patterns could have been done by building a string of the last several relevant characters and checking for the presence of each possible pattern every time a new character is received. However, a superior approach is to use a finite automaton to recognize the patterns, which requires keeping track of only the current state of the automaton. Implementation of finite automata for pattern recognition was rather simple. There is a labeled section of code for each state, a word holding the current state, and a dispatch table that contains pointers to the code for each state and is indexed by the value of the current state. The current state word could contain the actual pointer to the code for the current state, but the extra level of indirection added only a few words to the space requirements and it makes program modifications and testing much easier. The code for each state merely checks to see what the current character is, makes the appropriate state transition by changing the value of the current state word, and then takes the specified action, such as closing the MicroNOVA file. It then returns to the main body of the program. With careful programming, the end-of-line and the end-of-file patterns can be recognized by a single automaton. Thus, when a pattern character is encountered, the program simply jumps to the code for the current automaton state. The result is the appropriate action, if the pattern is complete, or an updated record of the status of the pattern recognition if not. This approach allows the pattern recognition process to be done in an easily modifiable way that does not complicate the main body of the program.

The character classification is done using the technique called spatially multiplexed character sets, which enables very fast answers to the question of whether or not a character is a member of a particular set of characters, such as the set of all characters that could be in a pattern to be recognized. Essentially, spatially multiplexed character sets are arrays of 128 words (for ASCII) in which each word represents an entry for a character and each bit position in a word represents the membership flag for a distinct character set. For example, the bits set in Figure 1 correspond to the situation where $\uparrow A$ and $\uparrow C$ are members of Set 0 , while $\uparrow A$ is a member of Set 2 and $\uparrow C$ is not. To find out if a given character is a member of a particular set, the character code is used as the index into the array, and a mask for

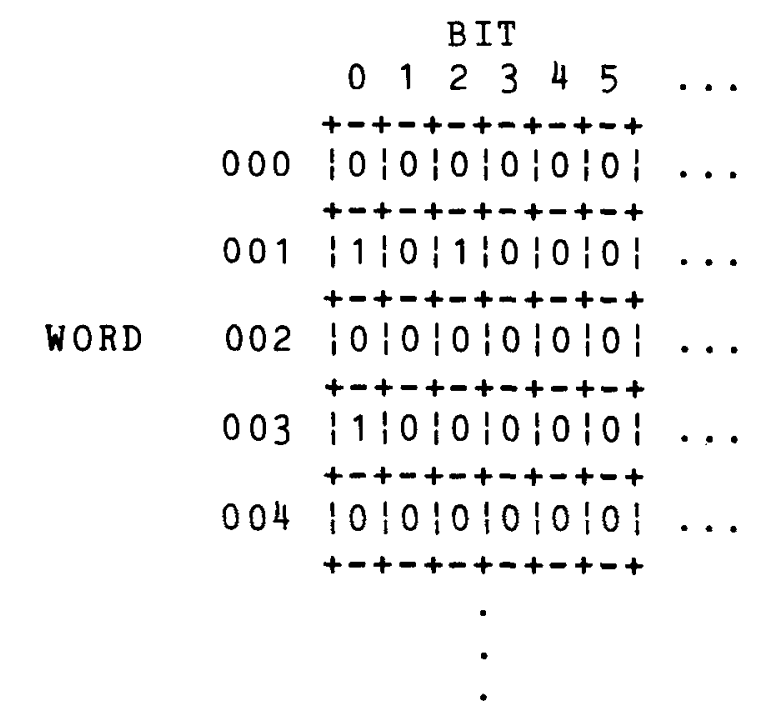

Figure 1. Illustration of spatially multiplexed character sets. An array of words, one for each character, contains 1 bit for each set that the character is a member of. 
the set in question is applied to that array entry. With this technique, the LINK10 program can rapidly determine whether a character requires special action.

Another feature that proved to be quite useful especially for program testing is the ability to translate all nonprinting characters being sent to the console to printable strings. This is implemented by providing a translate flag. This flag can be complemented at any time during program execution by typing a $\uparrow \mathrm{B}$ at the console. While the flag is set, all control characters (except $\langle\mathrm{CR}\rangle$ and $\langle\mathrm{LF}\rangle$ ) and $\langle\mathrm{DEL}\rangle$ are translated into an " $\uparrow$ " followed by a printable character.

In addition to being useful for testing, this feature helps to illustrate the power of spatially multiplexed character sets. Using a brute-force approach, the decision to translate or not translate a given character requires at least three range checks ([000,011], [013, 014], and [016,037]) and one character check (177). If at any time in the future, it was found desirable to change the set of characters that were translated, additional code would have to be written and more execution time spent checking each character when the translate flag is on. Using spatially multiplexed character sets, this decision can be made in one short piece of code whose size is independent of the set of characters to be translated, and that set can be modified merely by setting or clearing bits in the character set array.

\section{CONCLUSION}

The above is certainly not a complete description of the implementation of our virtual terminal program, and it certainly is not claimed to be the best way to solve all of the problems involved in the operation of such a program. However, it does show one highly effective way of attacking the problem of transferring files between a lab computer and a timesharing system. 